\title{
FS AUR AS A PERMANENT SUPERHUMP SYSTEM
}

\author{
Gaghik H. Tormassian' and Sergei I. Zharikor'
}

RESUMEN

\begin{abstract}
Descubrimos que la Variable Cataclismica FS Aur de corto periodo orbital muest ra periodo fotométrico cercano a orbital. El exceso de periodo coincide con lo que se espera de un sistema que cuenta con la presencia de superjorobas permanentes. Esto puede ser resultado de que FS Aur posea un disco grande y entable. Sin embargo el sistema sigue mostrando persistentemente un periodo mucho más largo que ol orbital y los nuevos datos no alteran las explicaciones previas de este periodo.
\end{abstract}

\section{ABSTRACT}

We discovered that the short period cataclysmic variable FS Aur at some epochs showis a photometric period close to the orbital. It exceeds the orbital period $b y \sim 2 \%$, which is a sign of the presinc of a permanent superhump in the system. Superhumps tend to appear near short, low amplitude outbursts. Wir assume that FS Aur possesses a large thermally stable accretion disc and that the outburst may be due to the variable mass transfer rate. This, however, does not alter our previous explanation of yet another. 2.4 times longer than orbital, photometric period of FS Aur, found earlier, and persistently observed in its light curves.

\section{Key Words: BINARIES: CLOSE - NOVAE, CATACLYSMIC VARIABLES — PHOTOMETRY}

\section{INTRODUCTION}

FS Aur is a short period Cataclysmic Variable (CV), with a spectroscopically established period of 85.7 minutes (Thorstensen et al., (1996), Neustroev(2002), Tovmassian et al., (2003)(T03)). It is on the short side of the Period Gap (PG) and, thus, is expected to have a mass ratio between components of $\mathrm{q} \leq 0.33$ suitable to produce superhumps as a result of an apsidal precession of the elliptically distorted accretion disk grown out of the resonance radius (3.1) (Whitehurst 1988). Must of the Dwarf Novae (DN) systems below the PG belong to the SU UMa subclass known to undergo superoutbursts, during which superhumps develop. Basically, there are two requirements that should be satisfied for the presence of superhumps: the above mentioned mass ratio and sufficiently large disc radius. While SU UMa stars reach the latter condition only during superoutburst, the theory that allows for the existence of systems that may have large enough discs in the quiescence and show so-called permanert superhumps. Not accidentally, more than 20 such systems were discovered in the last decade (Patterson 1999). The amplitudes of superhumps in these systems is highly variable and sometimes disappears altogether from the light curves. Osaki (1996) suggested that only the orbital period and mass transfer rate determine the behavior of the system, and thus its classification (Retter \& Naylor 2000). So far, FS Aur has

\footnotetext{
${ }^{1}$ Instituto de Astronomía, UNAM. Ensenada. México.
}

been never detected in superoutburst.

Another more bizarre peculiarity of FS Aur is that it shows a 205 min photometric period which more than two times excects its orbital period. After lengthy discussion of possible (auses of such peculiar behavior, T03 suggested a now possible mechanism to explain observed periods. It may be caused by the rapid rotating magnetic white dwarf (IVD) with a period of the order of 50 - 100 sece, which in addition precesses. According to existing models. the precession period for a rigidly rotating $I I D$ would be in order of the observed period. In order 10 see this precession period in optical warelengths, the collimated beam from the magnetically accreting white dwarf, the rotational axes of the white dwarf and the binary plane where the disc is located should have certain angles relative to each other. This could explain the uniqueness of FS Aur and why we do not observe such periods in other systemis. This hypothesis remains, however, highly debatable until firm evidence is not found of the fast rotating IVD in this system.

Motivated by this unique and unexplained behavior, we kept monitoring the star. Here we report on new findings suggesting that $F S$ S I ur is a permanent superhump system. which in turn may explain why we have never observed it in the superoutburst.

\section{PHO'TONETRY IND PERIODS}

A neit sot of observations of FS Aur using 1.jm telescope of OLN at SPXI was obtained on 13-15 

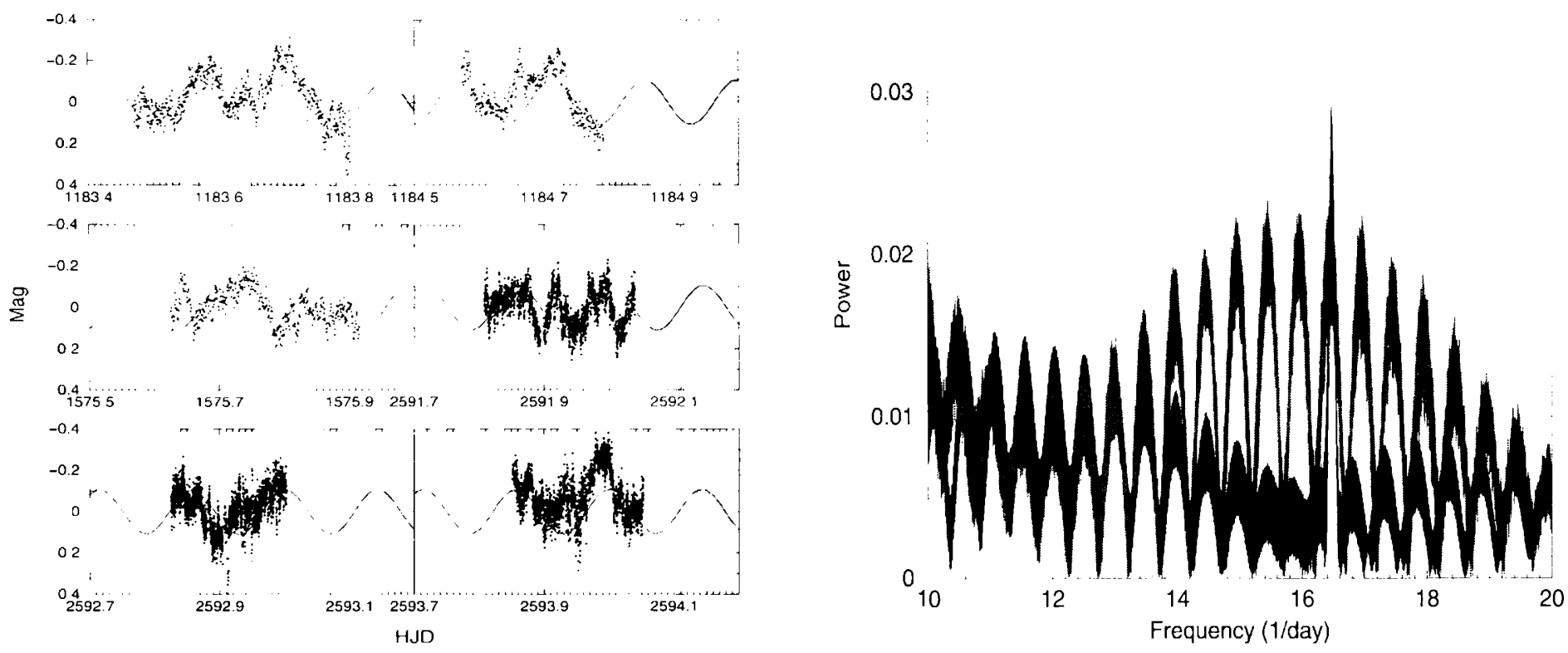

Fig. 1. The light curves of IS' Anl. Left pand: solection of nights where the superhump period appears (the last 3 sub-panels contain now (atai Right panel are nights where predominant period in the light curves is the long photometric period. The sin curve is a fit to the long photometric period over a 4 year period of monitoring.

Nox. 20102. The (' ('I) wiss binned $2 \times 2$ and trimmed in size to accommodate the objere and at least 2 comperison stars for a rapid differential photmetry in $\mathrm{R}$ band. The new light curves are highly variable and on first glance look sporadic. The clar sinusoidal matition with 205 min period observed before is replaced with a more complex pattern. However, the period search reverals a fow dominating frequencies. One of them remains the musterions long photometric period of 20.5 min. Another strong peak appears at the period close to the orbital. It is broad in the single night periodograms and it is difficult to tell if it coincides with the orbital period or not. Exen combining the data of 3 nights does not wat isfactorily resolve the emerging period. Therefore, we selected additioanal data from previons observations (T03). where similar variability was seen. In the Figure 1 on the left panel the selecterl observations are presented. including the new ones at the bot101n. In the right pancl the observations where the long photonetrie period (')arly dominates are presenterl for comparison. The Fourier transform on the seleretion of data presented in the left panel reverals multiple peaks due to the alias periods appearing be"ause of uneren distribution of data. The strongest peak drifts away from the value corresponding to the orbital period, loward longer periods. The (ILF.N procedure (Roberts et al. 1987) encounters the peak renterert on 16.46 day $^{-1}$. which comesponds to the $87.5 \mathrm{~min}$ period. The powor spectrum of the selected set of data. as well as its (LEA. Ved counterpart are presentere in Figure 2. Folding the data by periods corresponding to the peaks in the Power Spectrum also clearly favors the $87.5 \mathrm{~min}$ period. This period exceeds the orbital period of the system by $2.06 \%$.

There is an empirical relation between superhump period excess and the orbital period of superhump systems (Patterson 1998) supported by the theory (e.g. Nurray 2000). According to that relationship the $2 \%$ value of period excess is what one would expect for FS Aur with the orbital period of 85.7 minutes. This indirectly confirms our derived period. On the other hand, it explains why FS Aur has not been seen in the superoutburst. The systems that are found to lave permanent superhumps possess thermally stable large discs as a result of higher mass transfer rates than in SU UMa systems.

\subsection{Outbursts and long-term behavior}

The scenario discribed above would be not very surprising or rare at all if not for the history of cycli-

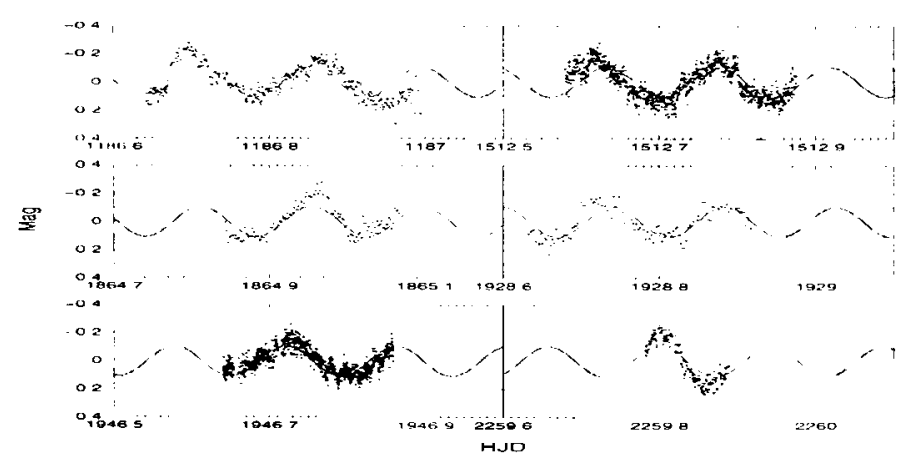

Fig. 2. The power spectrum of selected data (see text) and its CLEANed counterpart 
a

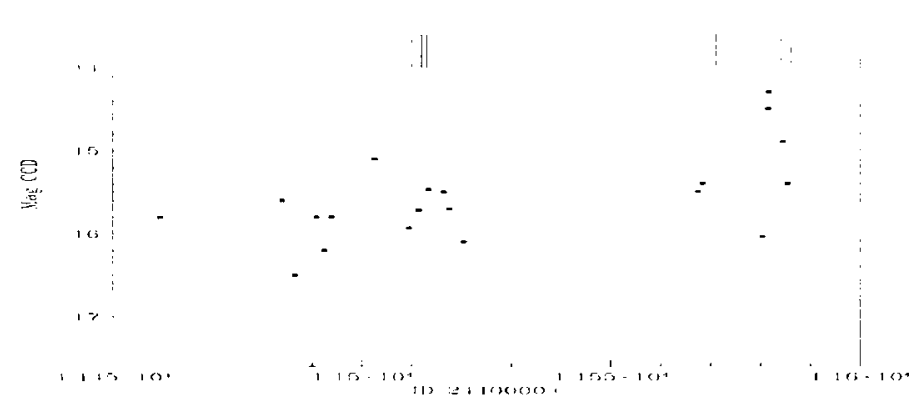

b

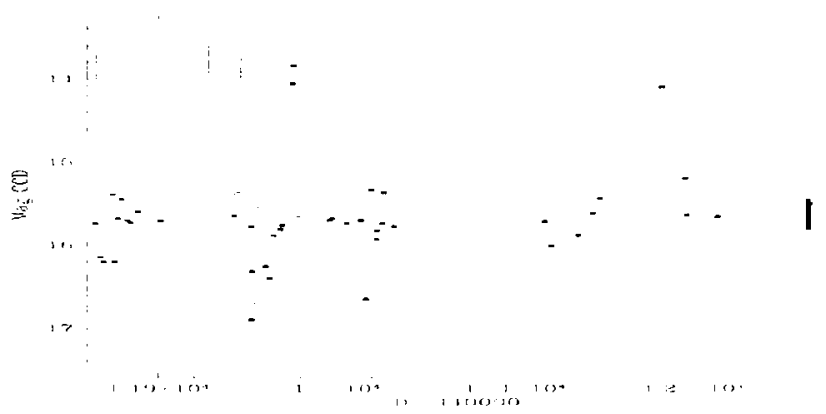

Fig. 3. The long-term light curve of FS Aur. The arrows mark the epochs whre we obtained high time resolution photometry. The thick arrows indicate nights when superhumps were dotecterl.

cal outbusrts of FS Aur. The problem is that the thermally stable disc in a high state should not produce outbursts in the way we used to explain them. The Dwalf Nova outbursts by widely accepted modols are a product of thermal instability and require lower mass transfer rate to maintain the temperature/viscosity in a range where a jump is possible from one stal, to another (Osaki 1989). WC examined all publicly available light curves of $\mathrm{FS}$ Aur from the archives of A AVSO. Only ('('I) measurements were taken into consideration. First wo checked the correlation with the occurence of the superhumps in the system with its long term behavior. In the Figure 3 the long-term light curve of FS Aur is presented from AAVSO archives. The dates when superhumps were observed in the light curves are marked by long arrows. The nights when light curves were predominantly displaying the long photometric period without significant interference of short period modulation are marked by small arrows. Whilc clatia is scarse and it is difficult to culant ify the overall impression is that superhumps mostly occur near/around the outbursts. If we amplify the outburst profile as. in the Figure 4, we can note that the duration of the flare, its amplitude and shape do not exactly fit the description of Dwarf Nova outburts. The short DN outbursts last on average 4 days, have

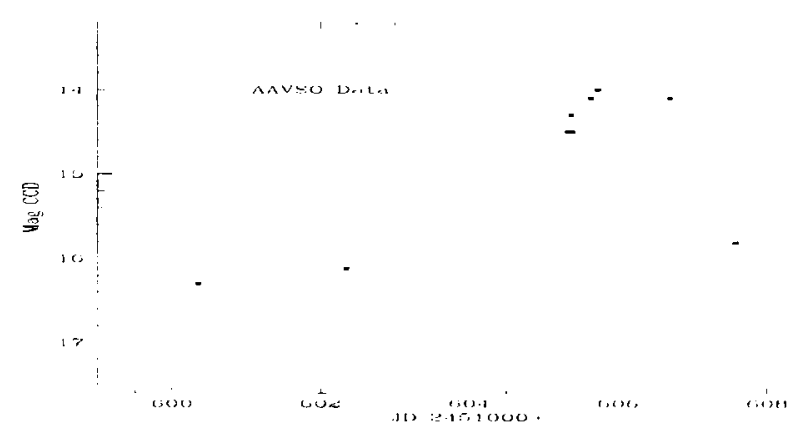

Fig. 1. The outburst profile of FS Aur. a stere rise. platean and slow decline and generally leach larger amplitudes (Narnor 1990). Whilo some frequent outbust systens ('xist (ER U Ma type: hato d Fumjaya 1995). FS Aur by no moans belongs to them. The quicserence of FS Aur lasts long enough to accumulate power for a descent I) $\mathrm{N}$ outhmist.

Wo suppose that the flares (as opposed to the outbursts in 1) $\mathrm{N}$ ) in Fis Aure rather a result of a varying mass transfer rate. which maly (anlse the observed pattern. There rxists a large number of models trying to oxplain I) N outbursts by a variable mass transfer ate or its combination with thermal instability model (eg Bath ot al. 195:3. Snak 2000). In such case. we can also argue that the increased mass transfer pushes the disc in the FS Aur beyond resonance radius limits and it starts to apsidally precess, producing the permanent superhumps.

Wr acknowledge with thankis the varialsle stall observations from the AAVSO International Database contributed by obsereres worldwiele and used in this research. Whe are gratelul to (') NAc'rT grants $34521 \mathrm{E}, 36585 \mathrm{E}$ y DGAPA grant IN 110002 .

\section{REFERENC'ES}

Bath, G. T., van Paradijs. J. 198:3. Natur. 305. 33

Kato, T., Kunjaya. C.. 1995. P.S.J. 47. 163

Murray.J., 2000, NINRAS. 31 1. L1

Neustroev. V.V. 2002, Ad.A. 322. .974

Osaki, Y., 1989. PASJ, 41, 1005

Osaki, Y., 1996, PASP, 10ऽ. 39

Patterson, J., less. PASP. 110, 1132

Patterson. J.. 1999. in "Disk Instabilities in ( lene Binary Systems". ed. Mineshige. Wheeler. L nir Acad. Press Retter. A.: Naylor. T. 2000. MIXR.1. :31!. 510

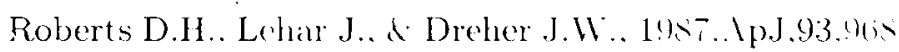
Smak, J., 2000, New Astr. Rev. 44, 171

Thorstensen. J.R. ot al. P'ASP. 108, - .3

'Tormassian, G.. et al., P.lsil' 115, 7.25 (TO3)

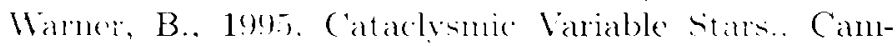
bridgo lniv. P'ress. ('ambridge

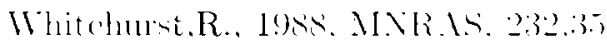

